\title{
EL EFECTO DEL FORMATO DE INTERVENCIÓN INDIVIDUAL O GRUPAL EN LA ESTIMULACIÓN COGNITIVA DE PACIENTES CON ENFERMEDAD DE ALZHEIMER
}

\author{
BERNARDino FERNÁNDEZ-CALVO ${ }^{1}$, ISRAEL CONTADOR ${ }^{2}$, AdRIANA SERNA ${ }^{2}$, Virginia \\ MENEZES DE LUCENA ${ }^{3}$ Y FRANCISCO RAMOS ${ }^{2}$ \\ ${ }^{1}$ Departamento de Psicología, Universidad Federal de la Paraíba, Brasil \\ ${ }^{2}$ Facultad de Psicología, Universidad de Salamanca, España \\ ${ }^{3}$ Departamento de Medicina Interna. Universidad Federal de la Paraíba, Brasil
}

\begin{abstract}
Resumen: El estudio trata de comprobar si el formato de la intervención individual o grupal, aplicado en un programa multimodal de estimulación cognitiva (PMEC), repercute en la evolución y las alteraciones de la conducta asociadas a pacientes con enfermedad de Alzheimer (EA). 45 participantes diagnosticados de EA probable fueron distribuidos aleatoriamente en 3 grupos. El rendimiento de los grupos experimentales, que recibieron el PMEC, en formato individual o grupal, fue comparado con un grupo de control, siguiendo un diseño pre-post, en una serie de medidas neuropsicológicas, funcionales y conductuales estandarizadas. Los grupos sometidos al PMEC lograron enlentecer, significativamente, el avance del deterioro cognitivo y reducir los síntomas conductuales de la EA. No obstante, la condición formato individual obtuvo mayores beneficios que la condición formato grupal. El formato de intervención individual, en comparación con el formato grupal, incrementa los beneficios que se derivan de la aplicación de un PMEC para pacientes con EA.
\end{abstract}

Palabras Clave: Demencia, enfermedad de Alzheimer, neuropsicología, intervención, estimulación cognitiva.

\section{The effect of an individual or group intervention format in cognitive stimulation of patients with Alzheimer's disease}

\begin{abstract}
The study aimed to verify whether the format of an individual or group intervention, applied in a multimodal cognitive stimulation program (MCSP), had an impact on progression and behavioral disturbances associated with Alzheimer's disease patients (AD). Forty-five participants diagnosed with probable $\mathrm{AD}$ were randomly divided into 3 groups. The performance of both experimental groups, receiving the MCSP either in individual or in group format, were compared with a control group with the use of a pre-post design on standard neuropsychological, functional and behavioral measures. Both MCSP groups achieved to significantly slow the progression of cognitive impairment and reduce behavioral symptoms associated with AD patients. However, the individual intervention format increases the benefits of the MCSP for AD patients in comparison with the group format.
\end{abstract}

Keywords: Dementia, Alzheimer's disease, neuropsychology, intervention, cognitive stimulation.

\section{INTRODUCCIÓN}

La falta de un tratamiento farmacológico definitivo, que permita curar y/o prevenir la

Recibido: 2-junio-2010; Aceptado: 12-julio-2010

Correspondencia: Francisco Ramos Campos, Facultad de Psicología, Universidad de Salamanca, Avda. De la Merced 109-131, 37005 Salamanca, España.

Correo-e: frc@usal.es

Agradecimientos: A la Asociación de Familiares de enfermos de Alzheimer de Salamanca (AFA). enfermedad de Alzheimer (EA), ha generado un creciente interés sobre las intervenciones que ayudan a optimizar el funcionamiento cognitivo y mejoran el ajuste emocional de éstas personas (Clare, 2008a). Así, las intervenciones centradas en aspectos cognitivos han demostrado que pueden ayudar a enlentecer la progresión del deterioro (Gatz et al., 1998; Sitzer, Twamley y Jeste, 2006; Woods, 2002) y reducir las alteraciones de conducta asociadas a la demencia (Olazarán et al., 2004; Spector et al., 2003), lo 
cual contribuye a mejorar la calidad de vida de los pacientes y sus familias (Zarit y Edwards, 1996).

No obstante, los resultados encontrados sobre los posibles beneficios de los programas de intervención, centrados en aspectos cognitivos, han sido a veces poco satisfactorios. Por ejemplo, Koltai, Welsh-Bohmer y Schmechel (2001) indicaron que el programa integrado de intervención en memoria y afrontamiento no tuvo efectos significativos sobre la cognición y el estado de ánimo subjetivo de los pacientes. También, Clare et al. (2003), tras analizar diferentes ensayos aleatorios controlados, concluyeron que el entrenamiento cognitivo no tenía efecto significativo alguno sobre las variables dependientes analizadas (cognitivas y conductuales).

Esta aparente falta de eficacia puede relacionarse con las limitaciones metodológicas que presentan los estudios en este campo. Aspectos tales como la falta de grupos de control, la asignación aleatoria de los sujetos y el uso de criterios amplios de inclusión han sido señalados por algunos autores (Chapman, Weiner, Rackley, Hynan y Zientz, 2004). No obstante, parece que las dificultades de generalización de las tareas cognitivas entrenadas a la vida diaria es uno de los problemas más destacados en la aplicación de programas basados en aspectos cognitivos (Clare y Woods, 2004). En cierto modo, los factores que repercuten en los beneficios logrados por los programas centrados en aspectos cognitivos están aún por descifrar (Koltai y Branch, 1999; Koltai et al., 2001; Van der Linden, Juillerat y Delbeuck 2004). Así, los programas de estimulación cognitiva (EC) han demostrado efectos positivos en etapas moderadas de la demencia (Spector et al., 2003), pero sus efectos en estadios leves han sido escasamente abordados (Breuvil et al., 1994).

Más concretamente, el formato de intervención empleado ha generado cierta controversia. Si bien compartir estrategias de afrontamiento en grupo puede reducir las emociones negativas asociadas al deterioro cognitivo (Wilson y Moffat, 1992; Zarit, Zarit y Rever, 1982), los enfoques individuales, centrados en la persona, consideran mejor las necesidades individuales y las reacciones emocionales de los pacientes con demencia (Díaz y Peraita, 2008; Kitwood, 1997). De cualquier modo, en la medida que el formato generalmente asumido en la $\mathrm{EC}$ ha sido el grupal (Clare y Woods, 2004), no existen estudios sistemáticos que comparen la posible interacción del formato con el programa de EC utilizado.

A diferencia de los programas de entrenamiento cognitivo (p.ej., basados en técnicas estandarizas y estructuradas por nivel de dificultad), hemos diseñado un programa asentado bajo un principio multimodal (Burgener, Yang, Gilberg y Marsch-Yant, 2008). Por un lado, las actividades tratan de estimular múltiples dominios cognitivos de una manera global (Luria, 1966); complementariamente se realizan una serie de actividades recreativas cuyo propósito es reducir el impacto del deterioro cognitivo en la vida emocional del sujeto (Farina et al., 2006; Karlsson, Brane, Melin, Nyth y Rybo, 1988).

El objetivo del presente estudio fue analizar si los pacientes con EA leve logran beneficiarse de la aplicación de un programa de EC multimodal. Particularmente, el estudio trata de analizar qué tipo de formato, individual frente al formato grupal, es el que logra mayores beneficios a nivel cognitivo, conductual $\mathrm{y}$ funcional.

\section{MÉTODO}

\section{Participantes}

Todos los participantes del estudio fueron seleccionados en la Asociación de Familiares de Enfermos de Alzheimer de Salamanca (AFA; Salamanca). La muestra total está compuesta por 45 pacientes que cumplían con los criterios del National Institute of Neurological and Communicative Disorders and StrokeAlzheimer's Disease and Related Disorders Association (NINCDS-ADRDA; McKhann et al., 1984) para EA «probable». Todos los pacientes, que recibían tratamiento con anticolinesterásicos, se encontraban en una fase leve de demencia (CDR 1; Hughes, Berg, Danziger, Cohen y Martín, 1982) y obtuvieron una pun- 
Tabla 1. Características sociodemográficas de los grupos. Medias (DT), y distribución según género

\begin{tabular}{lccc}
\hline & $E A I(n=15)$ & $E A G(n=15)$ & $E A N T(n=15)$ \\
\hline Edad & $75,53(4,71)$ & $75,13(5,08)$ & $75,33(4,49)$ \\
Género (Hombre/mujer) & $10 / 5$ & $7 / 8$ & $8 / 7$ \\
Escolaridad (años) & $7,20(2,24)$ & $8,00(3,02)$ & $6,93(3,53)$ \\
Mini-Mental State Examination & $18,33(2,46)$ & $19,06(3,01)$ & $19,53(1,84)$ \\
\hline
\end{tabular}

Nota. EAI: grupo de pacientes con estimulación individual; EAG: grupo de pacientes con estimulación grupal; EANT: Grupo de personas con EA en lista de espera.

tuaron inferior o igual 24 puntos en el MiniMental State Examination (MMSE, Folstein, Folstein, y McHugh 1975). Ninguno de ellos presentaba problemas lingüísticos, sensoriales $\mathrm{y} /$ motores que pudieran afectar a la aplicación de las pruebas. Tras obtener el consentimiento informado, del tutor legal o familiar más próximo, cada paciente fue asignado, aleatoriamente, a una de las siguientes condiciones: (a) EAI; pacientes que recibieron $\mathrm{EC}$ en el formato individual. (b) EAG; pacientes sometidos a un programa de EC en sesiones grupales. (c) EANT; pacientes que quedaban en lista de espera para recibir el mismo programa de intervención tras la finalización del estudio. Los grupos analizados no mostraron diferencias significativas en las variables estudiadas, clínicas y socio-demográficas, en la fase pretratamiento (ver Tabla 1).

\section{Instrumentos de evaluación}

Todos los participantes fueron evaluados por un neuropsicólogo experto en el ámbito de las demencias. Se utilizaron los siguientes instrumentos:

Mini-Mental State Examination (MMSE; Folstein et al., 1975). Versión española de Blesa et al. (2001). Es una prueba breve de cribado utilizada para evaluar globalmente el estado mental del paciente (rango puntuación $=0-30$ puntos). Las puntuaciones inferiores a 24 puntos indican un deterioro patológico de la función cognitiva.

Escala para la Evaluación de la Enfermedad de Alzheimer, subescala cognitiva
(ADAS-Cog, Mobs, Rosen y Davis, 1983). Versión española de Peña-Casanova et al. (1997). Está formada por 11 pruebas (atención, memoria, lenguaje, praxis y otras funciones cognitivas) diseñadas para evaluar el deterioro cognoscitivo en la EA. Las puntuaciones oscilan entre 0 y 70 puntos, de forma que a mayor puntuación más deterioro.

Inventario Neuropsiquiátrico Breve (NPI-Q; Kaufer et al., 2000). Versión española de Boada, Cejudo, Tárraga, López y Kaufer (2002). Es un instrumento clínico breve, que evalúa hasta 12 de los síntomas psicológicos y del comportamiento típicamente asociados a la demencia. Se obtienen puntuaciones sobre gravedad del síntoma (rango: $1=$ leve, $2=$ moderado, 3 = grave) y agotamiento emocional provocado en el cuidador (rango $=0-5$ ).

Escala de Depresión de Cornell (EDC; Alexopoulos, Abrams, Young y Shamoian, 1988). Versión española de Pujol-Doménech, de Azpiazu, Salamero y Cuevas (2001). Es un instrumento diseñado, específicamente, para la evaluación de la sintomatología depresiva en pacientes con deterioro cognitivo. Consta de 19 ítems agrupados en cinco dimensiones (estado ánimo, trastorno de conducta, síntomas físicos, funciones cíclicas y trastorno del pensamiento), que evalúan la intensidad de los síntomas depresivos (ausente, leve o intermitente y grave).

Escala de Evaluación Rápida de Discapacidad-2 (RDRS-2; Linn y Linn, 1982). Versión española de Monllau et al. (2006). Es una escala multidimensional para la evaluación rápida de la discapacidad en pacientes ancianos. Consta de 18 preguntas clasificadas en tres subesca- 
las: actividades cotidianas, grado de incapacidad (en comunicación, audición y visión) y problemas especiales de tipo neuropsiquiátrico (confusión, falta de cooperación y depresión). El rango de puntuación oscila entre 18-74 puntos, de tal forma que a mayor puntuación mayor discapacidad.

\section{PROCEDIMIENTO}

La evaluación fue realizada por el mismo examinador una semana antes y después de la intervención. El examinador fue ciego respecto a la condición a la que quedó asignado cada paciente. Todos los pacientes recibieron el mismo programa de intervención (tres meses $\times$ tres sesiones semanales $\times$ una hora sesión; total $=$ 36 sesiones). Las sesiones (individual o grupal) fueron realizadas por personal especializado en el manejo de pacientes con demencia. Una vez concluida la intervención los pacientes del grupo control (grupo en lista de espera) también recibieron el PMEC. El programa de EC estaba estructurado en cuatro momentos: (1) saludo inicial; (2 recuerdo de las actividades realizadas la sesión anterior; (3) desarrollo de la nueva actividad; y (4) evaluación de la sesión de trabajo. En cada sesión de trabajo se estimulaban al menos dos funciones cognitivas (p.ej., atención y memoria). Finalmente, y de forma complementaria, se realizaban diferentes actividades de ludoterapia. Todas las actividades realizadas en el programa se resumen en la Tabla 2.

\section{Análisis estadísticos}

La estadística descriptiva (media y $D T$ ) se utilizó para mostrar las características de los grupos en las variables analizadas. Un ANOVA de un solo factor permitió contrastar la existencia de diferencias significativas entre los grupos. Para comprobar el efecto del programa se empleó un ANOVA mixto de medidas repetidas de $3 \times 4$. El factor intra-sujeto corresponde con las variables dependientes (ADAS-Cog, NPI-Q, EDC y RDRS-2), mientras que el factor inter-sujetos viene determinado por los grupos (EAI, EAG y EANT). La significación general adoptada para el error tipo I fue $p<0$, 05 Las diferencias simples entre los tratamientos fueron ajustadas según la corrección de

Tabla 2. Actividades desarrolladas en el programa integrado de estimulación cognitiva

\begin{tabular}{|c|c|}
\hline Estimulación & Actividades \\
\hline Atención & $\begin{array}{l}\text { Descifrar mensajes utilizando un determinado código; ordenar las cartas de la baraja, contar } \\
\text { elementos; buscar letras de un texto determinado, entender el significado y recordar lo que ha } \\
\text { leído; encontrar diferencias; encontrar elemento sin emparejar; seguir ritmos, dar órdenes, contar } \\
\text { letras de un texto. }\end{array}$ \\
\hline Memoria & $\begin{array}{l}\text { Categorización, ordenar las palabras por grupos; ordenar palabras para formar frases; hacer } \\
\text { historias; reglas nemotécnicas; asociación; ordenar letras para formar palabras; fonética (palabras } \\
\text { que comienzan por determinadas sílabas, que contengan determinadas letras); recordar números; } \\
\text { memorizar los objetos contenidos en una lámina; copiar dibujos; comprensión de lectura a través } \\
\text { de preguntas sencillas. }\end{array}$ \\
\hline Cálculo & $\begin{array}{l}\text { Ordenar cifras de menor a mayor; resolución de problemas; series; operaciones aritméticas; } \\
\text { lectura de números; ordenar números. }\end{array}$ \\
\hline $\begin{array}{l}\text { Funciones } \\
\text { ejecutivas }\end{array}$ & $\begin{array}{l}\text { Juego del solitario; juego con preguntas de si o no; consecuencias; solucionar problemas; secuen- } \\
\text { cias; identificar causas. }\end{array}$ \\
\hline $\begin{array}{l}\text { Actividad } \\
\text { recreativa }\end{array}$ & $\begin{array}{l}\text { Juego de las palabras encadenadas: decir una palabra que empiece por la sílaba en que acabó la } \\
\text { palabra que dijo el usuario anterior; juegos de mesa adaptados a la EA; juego de pasar palabra; } \\
\text { palabras encadenadas, veo-veo. }\end{array}$ \\
\hline
\end{tabular}


Tabla 3. Rendimiento de los grupos (media y desviación típica) a nivel cognitivo, funcional y conductual: contrastes.

\begin{tabular}{|c|c|c|c|c|}
\hline & $E A I(n=15)$ & $E A G(n=15)$ & $\operatorname{EANT}(n=15)$ & Post-hoc \\
\hline ADAS-Cog pre & $23,06(1,16)$ & $23,33(3,08)$ & $23.20(2,90)$ & n.s \\
\hline ADAS-Cog post & $23,60(1,24)$ & $27,80(4,14)$ & $33,20(3,14)$ & $a^{* *}, b^{* *}, c^{*}$ \\
\hline NPI-Q pre & $6,00(2,59)$ & $6,13(1,99)$ & $6,20(2,73)$ & n.s \\
\hline NPI-Q post & $4,80(3,21)$ & $5,80(1,97)$ & $11,00(2,80)$ & $a^{* *}, b^{*}$ \\
\hline EDC-pre & $7,67(4,03)$ & $7,80(2,81)$ & $7,67(1,80)$ & n.s \\
\hline EDC-post & $5,53(3,36)$ & $6,93(3,65)$ & $12,13(2,53)$ & $a^{* *}, b^{*}, c^{*}$ \\
\hline RDRS-2-pre & $29,07(3,92)$ & $28,732(3,712)$ & $29,00(3,14)$ & n.s \\
\hline RDRS-2-post & $30,93(5,15)$ & $30,47(2,82)$ & $37,27(2,02)$ & $\mathrm{a}^{\dagger}$ \\
\hline
\end{tabular}

Nota. ADAS-Cog: Escala de Evaluación de la Enfermedad de Alzheimer; NPI-Q: Inventario Neuropsiquiátrico-Questionario; EDC: Escala Depresión Cornell; RDRS-2; Escala de Evaluación Rápida de Discapacidad-2; a: Diferencias ENT y EAI; b: Diferencias ENT y EAG, c: Diferencias EAI y EAG; ${ }^{*} p<0.05 ; * * p<0.01 ; \dagger$ Diferencia marginal.

Bonferroni. Todos estos análisis fueron realizados con el paquete estadístico SPSS 15.0 para Windows.

\section{RESULTADOS}

En la Tabla 3, aparece el rendimiento de los grupos (media y $D T$ ), durante las fases pre y post-tratamiento, en cada una de las variables dependientes seleccionadas. Los resultados del ANOVA mostraron que no había diferencias significativas entre los grupos, durante la fase pre-tratamiento, ni a nivel cognitivo (ADAS$\mathrm{Cog}$ ), ni funcional (RDRS-2), ni tampoco neuropsiquiátrico (NPI-Q y EDC) (en todos los casos $F<1$ ).

\section{$A D A S-C o g$}

El efecto del grupo resultó significativo en post-tratamiento $\left(F_{(2,42)}=4,66, p<0,001\right)$. Los contrastes a posteriori indicaron que los grupos de tratamiento EAI y EAG puntuaron significativamente más bajo que el grupo EANT. Ade- más, el grupo EAI puntuó significativamente mejor que EAG. El efecto del factor $\left(F_{(1,42)}=\right.$ $129,99, p<0,001)$ y de la interacción (ADAS$\operatorname{Cog} \times$ Grupo $)$ fueron significativos $\left(F_{(2,42)}=39\right.$, $20, p<0,001)$. De modo que, el grupo EAI mostró un declive cognitivo significativamente menor en comparación con los grupos EAG y EANT. La Figura 1 muestra el rendimiento de los grupos en ADAS-Cog antes y después del tratamiento.

\section{Inventario Neuropsiquiátrico breve (NPI-Q)}

Existieron diferencias significativas entre los grupos en la fase post-tratamiento $\left(F_{(2,42)}=10,04, p<0,001\right)$. Los contrastes a posteriori indicaron que los grupos experimentales EAI y EAG puntuaron significativamente mejor que EANT, pero no hubo diferencias significativas entre EAI y EAG. Finalmente, el efecto del factor $\left(F_{(1,42)}=5,68, p<0,05\right)$ y de la interacción $(\mathrm{NPI}-\mathrm{Q} \times$ grupo) resultaron significati$\operatorname{vos}\left(F_{(2,42)}=16,81, p<0,001\right)$. En consecuencia, los grupos EAI y EAG mostraron una reducción significativa de los SPCD de la demencia en 


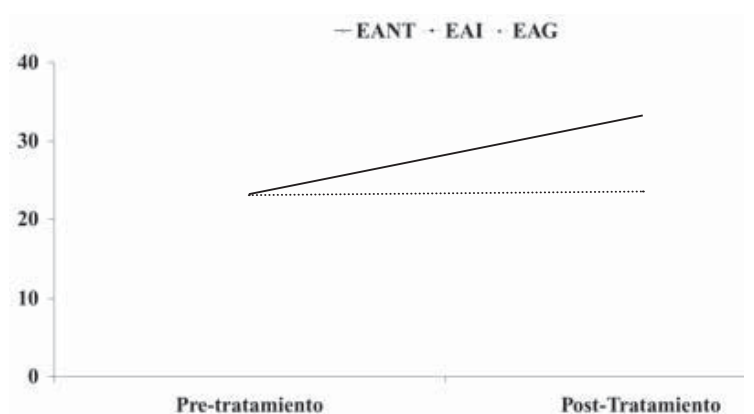

Figura 1. Cambios en las puntuaciones pre- y post-tratamiento en la Escala de Evaluación de la Enfermedad de Alzheimer, según los grupos (EANT, EAI y EAG).

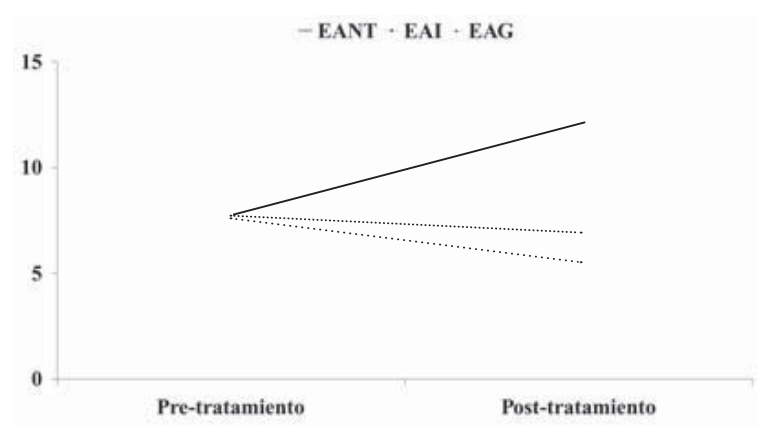

Figura 3. Cambios en las puntuaciones pre- y posttratamiento en la Escala de Cornell, según los grupos (EANT, EAI y EAG).

relación a EANT. La Figura 2 muestra el rendimiento de los grupos en el NPI-Q antes y después del tratamiento.

\section{Escala de depresión de Cornell (EDC)}

El efecto principal del grupo sobre la EDC fue significativo en post-tratamiento $\left(F_{(2,42)}=\right.$ 5,96, $p<0,01)$. Los contrastes a posteriori indicaron que los grupos EAI y EAG obtuvieron una puntuación significativamente inferior a EANT. El grupo EAI puntuó significativamente más bajo que EAG. El efecto del factor $\left(F_{(1,42)}\right.$ $=11,21, p<0,05)$ y de la interacción de la medida EDC con el grupo fueron significativos $\left(F_{(2,42)}=20,71, p<0,001\right)$. Así, el grupo EAI mostró una reducción significativamente mayor de los síntomas depresivos en comparación con los otros grupos. La Figura 3 muestra el rendimiento de los grupos en la EDC antes y después del tratamiento.

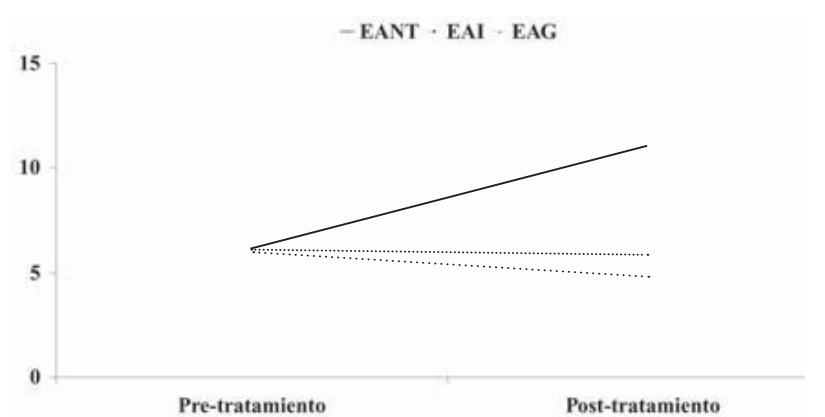

Figura 2. Cambios en las puntuaciones pre- y posttratamiento en el Inventario Neuropsiquiátrico Breve, según los grupos (EANT, EAI y EAG).

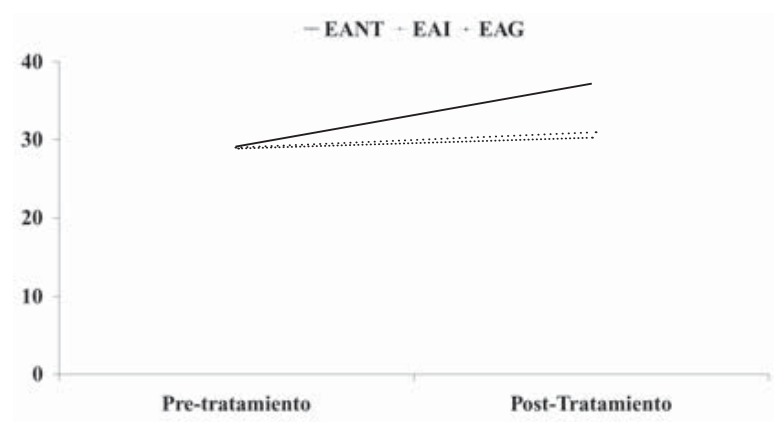

Figura 4. Cambios en las puntuaciones pre- y posttratamiento en la Escala de Evaluación Rápida de Discapacidad-2, según los grupos (EANT, EAI y EAG).

\section{Escala funcional (RDRS-2)}

El efecto principal del grupo fue significativo post-tratamiento $\left(F_{(2,42)}=4,94, p=0,012\right)$. Así, los contrastes a posteriori indicaron que el grupo EAI obtuvo una puntuación marginalmente inferior al grupo EANT $(p<0,05)$, pero no hubo diferencias significativas entre EAG y EANT, ni tampoco entre los grupos de tratamiento. El efecto del factor $\left(F_{(1,42)}=113,04, p<0\right.$, $001)$ y de la interacción (RDRS- $2 \times$ grupo) fueron significativos $\left(F_{(2,42)}=33,58, p<0,001\right)$. Así pues, a pesar que el declive funcional fue más lento para el grupo EAI, este descenso sólo obtuvo una significación marginal. La Figura 4 muestra el rendimiento de los grupos en la RDRS-2 antes y después del tratamiento.

\section{DISCUSIÓN}

Los resultados del estudio demuestran que el PMEC logró aminorar el declive cognitivo 
y mejorar el ajuste, psicológico y conductual, de los pacientes con EA. Éstos resultados son avalados por Spector et al. (2003), que demostraron que la estimulación cognitiva es una herramienta que produce beneficios sobre la cognición de los pacientes con demencia. También, Chapman et al. (2004) observaron que los pacientes que recibieron el programa de estimulación cognitiva combinado con donepezilo, en relación con el grupo que sólo recibió terapia farmacológica, no sólo mostraron una mejoría cognitiva sino también en la sintomatología emocional negativa.

Resulta interesante señalar que, los pacientes con EA que recibieron las sesiones en formato individual obtuvieron un declive cognitivo significativamente más lento, en comparación con los pacientes que recibían la estimulación en formato grupal. Del mismo modo, el grupo de estimulación individual mostró un reducción de la sintomatología depresiva significativamente mayor que el grupo de estimulación grupal.

El informe de Breuil et al. (1994) destaca que la estimulación cognitiva, en formato grupal, puede tener efectos positivos sobre la cognición de pacientes con EA leve y, además, sus efectos se pueden generalizar a la conducta (Spector, Orrell, Davies y Woods, 2007). Sin embargo, no se puede afirmar que el formato grupal sea apropiado para todos los pacientes del grupo, especialmente, cuando algunos pacientes tienen dificultades en seguir el desarrollo de cada sesión (Spector, Orrell, Davies y Woods, 2001). Este hecho puede explicar que la mejoría encontrada en el formato grupal sea inferior a la del individual, pues la eficacia del formato grupal homogénea en todos individuos. Paralelamente, Sitzer et al. (2006) sostienen que para la mejora de los síntomas cognitivos y funcionales asociados a la EA los programas de entrenamiento cognitivo individualizados, generalmente, son más eficaces que los grupales.

En contra de nuestros resultados, Kotai et al. (2001) encontraron que los pacientes sometidos al programa de memoria y afrontamiento, aplicado de manera individual o grupal, en comparación con el grupo control, no mostraron beneficio ni cognitivo ni conductual. Estos resultados se explican, fundamentalmente, por las limitaciones metodológicas y el impacto de la técnica de intervención. Aunque la capacidad de generalización de los programas de entrenamiento cognitivo a otras tareas ha sido cuestionada (Davis, Massman y Doody, 2001), la intervención multimodal empleada en nuestro trabajo parece potenciar la eficacia y la capacidad de generalización del mismo (Gates y Valenzuela, 2010).

Adicionalmente, el aumento del tamaño de la muestra y el número de sesiones en nuestro estudio, en comparación a otros estudios tales como los de Kotai et al. (2001) y Ávila et al. (2007), podría avalar la significación encontrada sobre las medidas cognitivas y conductuales que hemos analizado (Requena, Maestu, Campo, Fernández y Ortiz, 2006), Por contra, aunque el grupo de estimulación individual mostró una tendencia hacia la mejora en las actividades funcionales, el efecto del programa no resultó significativo en esta variable.

Resultados similares a los nuestros han sido obtenidos por Tárraga et al. (2006); los pacientes que recibieron la EC mostraron beneficios cognitivos, aunque el deterioro funcional de los pacientes permaneció intacto. Es posible que la medida funcional utilizada en nuestro estudio (RDRS-2) no fuera sensible a los cambios posttratamiento (Onder et al., 2002), concretamente, cuando todos los pacientes del estudio se encuentran en una fase leve de la demencia (rango MMSE: 18-24) y las actividades básicas están generalmente preservadas. Por otra parte, la tendencia encontrada en el grupo EAI, sobre la mejora en la actividad funcional, puede entenderse a partir de que la intervención individual satisface mejor las necesidades específicas (cognitivas y emocionales) del paciente (Clare, 2008b).

No obstante, nuestra investigación presenta algunas limitaciones. Por un lado, el tamaño de la muestra que puede parecer reducido y, por otro, la dificultad para separar los efectos de cada elemento del programa (cognición vs. actividad recreativa). Sin embargo, estos problemas son comunes en este tipo de intervenciones debido, sobre todo, a la dificultad de reunir un grupo homogéneo de pacientes. Además, el hecho de que ambos grupos de estimulación recibieran la actividad recreativa impide separar los efectos de la estimulación cognitiva, por un lado, y la actividad recrea- 
tiva, por otro. Pero, esto no fue contemplado como objetivo del estudio y la aplicación multimodal del programa dificultó este propósito.

A modo conclusión, parece que el PMEC es una herramienta útil de intervención en pacientes con EA. Específicamente, el estudio apoya el uso de éste tipo de programas en pacientes en estadios leves de la EA. Además, las personas con EA se benefician más de la intervención cognitiva individual que de la grupal. De éste modo, se aboga por el uso de una perspectiva de acción centrada en la persona, donde se atiendan sus dificultades cognitivas y emocionales de una manera específica. Por último, el presente trabajo supone un impulso para la investigación de variables relacionadas con los efectos de la EC en pacientes con EA. Además, son temas de reconocido interés para el presente y el futuro continuar investigando cómo ciertas variables, asociadas a los pacientes (p.ej., gravedad de la demencia) y al tipo de terapia empleado (duración sesiones, número, formato $\mathrm{y}$ actividades desarrolladas), afectan a los resultados obtenidos con este tipo de intervenciones.

\section{REFERENCIAS}

Alexopoulos, GS., Abrams, RC., Young, R.C., y Shamoian, C.A. (1988). Cornell scale for depression in dementia. Biological Psychiatry, 23, 271-284.

Ávila, R., Carvalho, I.A. M., Bottino, C.M.C., y Miotto, E. (2007). Neuropsychological rehabilitation in mild and moderate Alzheimer's disease patients. Behavioural Neurology 18, 225-233.

Blesa, R., Pujol, M., Aguilar, M., Santacruz, P., BertranSerra, I., Hernández, G., Sol, J.M. y Peña-Casanova, J. NORMACODEM Group (2001). Clinical validity of the 'mini-mental state' for Spanish speaking communities. Neuropsychologia, 39, 1150-1157.

Boada, M., Cejudo, J. C., Tárraga, L., López, O. L., y Kaufer, D. (2002). Neuropsychiatric Inventory Questionnaire (NPI-Q): Validación española de una forma abreviada del Neuropsychiatric Inventory (NPI). Neurología, 17, 317-323.

Breuil, V., de Rotrou, J., Forette, F., Tortrat, D., GanasiaGanem, A., Frambourt, A., Moulin, F., y Boller, F. (1994). Cognitive stimulation of patients with dementia: Preliminary results. International Journal of Geriatric Psychiatry, 9, 211-217.

Burgener, S.C., Yang, Y., Gilbert, R., y Marsh-Yant, S. (2008). The Effects of a Multimodal Intervention on
Outcomes of Persons with Early Stage Dementia. American Journal of Alzheimer's Disease and Other Dementias, 23, 382-394.

Chapman, S.B., Weiner, M.F., Rackley, A., Hynan, L. S., y Zientz, J. (2004). Effects of cognitive-communication stimulation for Alzheimer's disease patients treated with donepezil. Journal of Speech, Language, and Hearing Research, 47, 1149-1163.

Clare, L., Woods, R.T., Moniz-Cook, E.D., Orrell, M., y Spector, A. (2003). Cognitive rehabilitation and cognitive training interventions targeting memory functioning in early stage Alzheimer's disease and vascular dementia (Cochrane Review). En The Cochrane Library, Issue 4, 2003. Chichester, UK: John Wiley \& Sons Ltd.

Clare, L. (2008a). Introducing neuropsychological rehabilitation and people with dementia. En L. Clare (Ed.), Neuropsychological Rehabilitation and People with Dementia (pp. 1-4). Hove: Psychology Press.

Clare, L. (2008b). Towards individualized rehabilitation interventions. En L. Clare (Ed.), Neuropsychological Rehabilitation and People with Dementia (pp. 65-82). Hove: Psychology Press.

Clare, L., y Woods, R.T. (2004). Cognitive training and cognitive rehabilitation for people with early-stage Alzheimer's disease: A review. Neuropsychological Rehabilitation, 14, 385-401.

Davis, R., Massman, P., y Doody, R. (2001). Cognitive intervention in Alzheimer disease: A randomized placebo-controlled study. Alzheimer Disease and Associated Disorders, 15, 1-9.

Díaz, C., y Peraita, H. (2008). Neuropsychological evaluation and cognitive evolution of a bilingual Alzheimer patient. Revista de Psicopatología y Psicología clínica, 13(3), 219-228

Farina, E., Mantovani, F., Fioravanti, R., Rotella, G., Villanelli, F., Imbornone, E., Olivotto, F., Tincani, M., Alberoni, M., Petrone, E., Nemni, R., y Postiglione, A. (2006). Efficacy of recreational and occupational activities associated to psychologic support in mild to moderate Alzheimer disease: a multicenter controlled study. Alzheimer Disease and Associated Disorders, 20, 275-282.

Folstein, M.F., Folstein, S.E., y McHugh, P.R. (1975). Mini-Mental State: A practical method for grading the state of patients for the clinician. Journal of Psychiatric Research, 12, 189-198.

Gatz, M., Fiske, A., Fox, L., Kaskie, B., Kasl-Godley, J.E., McCallum, T.J., y Wetherell, J.L. (1998). Empirically validated psychological treatments for older adults. Journal of Mental Health and Aging, 4, 9-45.

Gates, N., y Valenzuela, M. (2010). Cognitive exercise and its role in cognitive function in older adults, Current Psychiatry Reports, 12, 20-27.

Hughes, C.P., Berg L., Danziger W, L., Cohen L.A., y Martin, R. (1982). A new clinical scale for the staging of dementia. British Journal of Psychiatry, 140, 566-572. 
Karlsson, I., Brane, G., Melin, E., Nyth, A.I., y Rybo, E. (1988). Effects of environmental stimulation on biochemical and psychological variables in dementia. Acta Psychiatrica. Scandinavica, 77, 207-213

Kaufer, D.I., Cummings, J.L., Ketchel, P., Smith, V., MacMillan, A., Shelleu, T., López, O.L., y DeKosky, S.T. (2000). Validation of the NPI-Q, a brief clinical form of the Neuropsychiatric Inventory. Journal of Neuropsychiatry \& Clinical Neurosciences, 12, 233-239.

Kitwood, T. (1997). Dementia reconsidered: The person comes first. Buckingham: Open University Press.

Koltai, D.C., y Branch, L.G. (1999). Cognitive and affective interventions to maximise abilities and adjustment in dementia. Annals of Psychiatry, 7, 241-255.

Koltai, D.C., Welsh-Bohmer, K.A., y Schmechel, D.E. (2001). Influence of anosognosia on treatment outcome among dementia patients. Neuropsychological Rehabilitation, 11, 455-475.

Linn, M.W., y Linn, B.S. (1982). The rapid disability rating scale. Part 2. Journal of the American Geriatrics Society; 139, 1136-1139.

Luria, A.R. (1966). Human Brain and Psychological Processes. New York: Harper \& Row.

McKhann, G., Drachman, D., Folstein, M., Katzman, R., Price, D., y Stadlan, E.M. (1984). Clinical diagnosis of Alzheimer's disease: Report of the NINCDS-ADRDA work group under the auspices of the Department of Health and Human Services task force on Alzheimer's disease. Neurology, 34, 939-944.

Mobs, R.C., Rosen, W.G., y Davis, K.L. (1983). The Alzheimer's Disease Assessment Scale: An instrument for assessing treatment efficacy. Psychopharmacological Bulletin, 19, 448-450.

Monllau A., Aguilar M., Pena-Casanova J., Bohm P., Blesa R., Sol J.M., Hernández G., y grupo NORMACODEM (2006). Estudio de la Escala de Evaluación Rápida de Discapacidad-2 (Rapid Disability Rating Scale-2) en la enfermedad de Alzheimer: datos del proyecto NORMACODEM. Neurología, 21, 282-288.

Olazarán, J., Muñiz, R., Reisberg, B., Peña-Casanova, J., del Ser, T., Cruz-Jentoft, A. J., Serrano, P., Navarro, E., García de la Rocha, M. L., Frank, A., Galiano, M., Fernández-Bullido, Y., Serra, J. A., González-Salvador, M. T., y Sevilla, C. (2004). Benefits of cognitive motor intervention in MCI and mild to moderate Alzheimer's disease. Neurology, 63, 2348-2353.

Onder, G., Penninx, B. W., Lapuerta, P., Fried, L.P., Ostir, G.V., Guralnik, J. M., y Pahor, M. (2002). Change in physical performance over time in older women: the Women's Health and Aging Study. Journal of Gerontology Series A: Biological Sciences and Medical Sciences, 57, 289-293.

Peña-Casanova, J., Aguilar, M., Santacruz, P., Bertrán Serra, I., Hernández, G., Sol, J.M., Pujol, A., Blesa, R. y grupo NORMACODEM (1997). Adaptación y normalización españolas de la Alzheimer's Disease As- sessment Scale (NORMACODEM) (y II). Neurología, 12, 69-77.

Pujol-Doménech, J., de Azpiazu, P., Salamero, M., y Cuevas, R. (2001). Sintomatología depresiva en la demencia. Escala de Cornell: validación de la versión en castellano. Revista de Neurología, 33, 397-398

Requena, C., Maestu, F., Campo, P., Fernández, A., y Ortiz, T. (2006). Effects of cholinergic drugs and cognitive training on dementia: 2-Year follow-up. Dementia and Geriatric Cognitive Disorders, 22, 339-345

Rosen, W.G., Mohs, RC., y Davis, K.L. (1984). A new rating scale for Alzheimer's disease. The American Journal of Psychiatry, 141, 1356-1364.

Sitzer, D.I., Twamley, E.W., y Jeste, D.V. (2006). Cognitive training in Alzheimer's disease: a meta-analysis of the literature. Acta Psychiatrica Scandinavica, 114, 75-80.

Spector, A., Orrell, M., Davies, S., y Woods, R.T. (2007). Reality orientation for dementia. Cochrane Database of Systematic Reviews (Issue No. 3), CD001119.

Spector, A., Orrell, M., Davies, S., y Woods, B. (2001). Can Reality Orientation be rehabilitated? Development and piloting of an evidence-based programme of cognition-based therapies for people with dementia. Neuropsychological Rehabilitation, 11, 377-397.

Spector, A., Thorgrimsen, L., Woods, R. T., Royan, L., Davies, S., Butterworth, M., y Orrell, M. (2003). Efficacy of an evidence-based cognitive stimulation therapy programme for people with dementia. British Journal of Psychiatry. 183, 248-254.

Tárraga, L., Boada, M., Modinos, G., Badenas, S., Espinosa, A., Diego, S., Morera, A., Guitart, M., Balcells, J., López, O. L., y Becker, J. T. (2006). A randomized pilot study to assess the efficacy of Smartbrain ${ }^{\circledR}$, an interactive, multimedia tool of cognitive stimulation in Alzheimer's disease. Journal of Neurology, Neurosurgery, and Psychiatry, 77, 1116-1121.

Van der Linden, M., Juillerat, A.C., y Delbeuck, X. (2004). Cognitive rehabilitation in mild cognitive impairment and prodromal Alzheimer's disease. En S. Gauthier, P. H. Scheltens, y J. Cummings (Eds.), Alzheimer's disease and related disorders (pp. 81-96). London: Martin Dunitz Limited.

Wilson, B.A., y Moffat, N. (1992). The development of group memory therapy. En B. A. Wilson, y N. Moffat (Eds.), Clinical Management of Memory Problems (2nd ed., pp. 242-273). London: Chapman \& Hall.

Woods, R.T. (2002). Reality orientation: A welcome return?. Age and Ageing, 31, 155-156.

Zarit, S.H., y Edwards, A.B. (1996). Family caregiving: research and clinical intervention. En R.T. Woods (Ed), Handbook of the Clinical Psychology of Ageing (pp. 331-368). Oxford, England: John Wiley \& Sons.

Zarit, S.H., Zarit, J.M., y Reever, K.E. (1982). Memory training for severe memory loss: Effects on senile dementia patients and their families. Gerontologist, 22, 373-377. 